\title{
Columnar magnetic structure coupled with orthorhombic distortion in the antiferromagnetic iron arsenide $\mathrm{SrFe}_{2} \mathrm{As}_{2}$
}

\author{
K. Kaneko, ${ }^{1,2, *}$ A. Hoser, ${ }^{3}$ N. Caroca-Canales, ${ }^{1}$ A. Jesche,${ }^{1}$ C. Krellner, ${ }^{1}$ O. Stockert, ${ }^{1}$ and C. Geibel ${ }^{1}$ \\ ${ }^{1}$ Max-Planck-Institut für Chemische Physik fester Stoffe, Nöthnitzer Strasse 40, 01187 Dresden, Germany \\ ${ }^{2}$ Advanced Science Research Center, Japan Atomic Energy Agency, Tokai, Naka, Ibaraki 319-1195, Japan \\ ${ }^{3}$ Helmholtz-Zentrum Berlin für Materialien und Energie, Glienicker Strasse 100, 14109 Berlin, Germany
}

(Received 17 July 2008; revised manuscript received 7 November 2008; published 5 December 2008)

\begin{abstract}
Neutron powder-diffraction experiments were carried out on polycrystalline $\mathrm{SrFe}_{2} \mathrm{As}_{2}$ in order to determine the magnetic structure and its relationship with the crystallographic one. Below $T_{0}=205 \mathrm{~K}$, magnetic reflections appear simultaneously with the onset of the orthorhombic distortion. The magnetic propagation vector of $\mathrm{SrFe}_{2} \mathrm{As}_{2}$ is determined to be $\boldsymbol{q}=\left(\begin{array}{lll}1 & 0 & 1\end{array}\right)$; the coupling of Fe moments is antiferromagnetic along the longer $a$ direction within the Fe-As layer, and the interlayer coupling is antiferromagnetic as well. The size of the Fe magnetic moment is deduced to be 1.01(3) $\mu_{B}$ with an orientation parallel to the $a$ axis. The temperature dependence of the magnetic moment shows excellent agreement with not only that of the muon precession frequency but also with that of the structural distortion, revealing the strong coupling of the magnetic order and the structural distortion in $\mathrm{SrFe}_{2} \mathrm{As}_{2}$. The discrepancy in the deduced size of the ordered moment between neutron and Mössbauer and $\mu \mathrm{SR}$ may imply an unusual magnetism in $\mathrm{SrFe}_{2} \mathrm{As}_{2}$.
\end{abstract}

DOI: 10.1103/PhysRevB.78.212502

PACS number(s): 75.25.+z, 75.30.Fv, 75.40.Cx, 75.50.Ee

The discovery of superconductivity in doped LaFeAsO triggered intensive research on layered $\mathrm{FeAs}$ systems. ${ }^{1}$ So far, the superconducting (SC) transition temperature $T_{\mathrm{SC}}$ of $R \mathrm{Fe} A \mathrm{sO}$ has rapidly been raised up to $54 \mathrm{~K}^{2-6}$ Furthermore, a similar high $T_{\mathrm{SC}}$ exceeding $35 \mathrm{~K}$ was also discovered upon doping the related compounds $\left.A \mathrm{Fe}_{2} \mathrm{As}_{2}(A=\mathrm{Ca}, \mathrm{Sr}, \mathrm{Ba})\right)^{7,8}$ $A \mathrm{Fe}_{2} \mathrm{As}_{2}$ has the well-known $\mathrm{ThCr}_{2} \mathrm{Si}_{2}$-type structure, which can be regarded as replacing the $\left(\mathrm{R}_{2} \mathrm{O}_{2}\right)^{2+}$ layer in $R \mathrm{FeAsO}$ by a single divalent ion $\left(A^{2+}\right)$ layer keeping the same electron count. ${ }^{9} R \mathrm{FeAsO}$ and $A \mathrm{Fe}_{2} \mathrm{As}_{2}$ were found to present very similar physical properties. Undoped $R \mathrm{FeAsO}$ compounds $(R=\mathrm{La}-\mathrm{Gd})$ present a structural transition at $T_{0} \sim 150 \mathrm{~K}$ followed by the formation of a spin-density wave (SDW) at a slightly lower temperature $T_{N} \sim 140 \mathrm{~K}$. $A \mathrm{Fe}_{2} \mathrm{As}_{2}$ exhibits a similar structural distortion, whereas the SDW forms at the same or a slightly lower temperature. ${ }^{9-13}$ Electron or hole doping leads to the suppression of the SDW and to the onset of superconductivity. This connection between a vanishing magnetic transition and the simultaneous formation of a SC state is reminiscent of the behavior in the cuprates and in the heavy-fermion systems, suggesting the SC state in these doped FeAs systems to be of unconventional nature. In addition, the SDW state is strongly coupled to the lattice distortion in this system and therefore it is of high interest to reveal the relationship between the lattice, the magnetism, and superconductivity.

The magnetic structures of the FeAs system have been investigated by neutron-diffraction study on $R \mathrm{FeAs}(\mathrm{O}, \mathrm{F})$ for $R=\mathrm{La}, \mathrm{Ce}$, and $\mathrm{Nd}$, and $\mathrm{BaFe}_{2} \mathrm{As}_{2}{ }^{14-16}$ The antiferromagnetic (AFM) reflection was observed around the $(1,0)$ or $(0,1)$ position with respect to the $\mathrm{Fe}$-As layer. This corresponds to $\mathrm{Fe}$ moments with AFM coupling along the $a$ or $b$ direction called the columnar structure. In contrast, interlayer coupling is not unique among these compounds, suggesting a relatively weak coupling between the layers. The size of the estimated $\mathrm{Fe}$ moment is less than $1 \mu_{B}$, which is much smaller than initial theoretical predictions. The strong dis- crepancy could be clarified since the calculated Fe moment depends sensitively on the As positions and the used potential. ${ }^{17-19}$ So far the magnetic structures of these iron arsenides have not been determined uniquely; especially the relation of the direction of the AFM ordering with respect to the short or long Fe-Fe distances is not settled. This is one of the fundamental questions on the magnetic properties of these materials and is indispensable for understanding the interplay between magnetism and superconductivity.

Among the reported FeAs systems, $\mathrm{SrFe}_{2} \mathrm{As}_{2}$ should be a suitable compound to study the detailed magnetic structure. By the substitution of $\mathrm{Sr}$ with $\mathrm{K}$ or Cs, the superconductivity appears with the maximum $T_{\mathrm{SC}}$ of $38 \mathrm{~K} .^{7,8}$ Specific heat on high quality powder sample of $\mathrm{SrFe}_{2} \mathrm{As}_{2}$ reveals a first-order transition at $T_{0}=205 \mathrm{~K}$, where both small hysteresis and latent heat were observed. ${ }^{9}$ A single crystal of $\mathrm{SrFe}_{2} \mathrm{As}_{2}$ was also studied recently, which also shows one transition at slightly lower temperature of $T_{0}=196 \mathrm{~K} .{ }^{12}$ The coexistence of the low temperature and the high temperature phase was also observed for the single crystal and is consistent with the first-order transition. Detailed studies clarified that both SDW and the structural transition from tetragonal $(I 4 / \mathrm{mmm})$ to orthorhombic $(\mathrm{Fmmm})$ occur at $T_{0} \cdot{ }^{10,11}$ The stronger magnetism in $\mathrm{SrFe}_{2} \mathrm{As}_{2}$ is inferred from the higher ordering temperature, the larger value of the Pauli-type susceptibility above $T_{0}$, as well as the larger Fe hyperfine field observed in Mössbauer experiments. ${ }^{10,11}$ Therefore, a larger ordered moment is expected for this compound, which should allow a detailed analysis.

In this Brief Report, we report a neutron powderdiffraction study on $\mathrm{SrFe}_{2} \mathrm{As}_{2}$. The precise analysis of the observed magnetic Bragg peaks on $\mathrm{SrFe}_{2} \mathrm{As}_{2}$ allowed us to uniquely determine the magnetic structure. The magnetic propagation vector of $\mathrm{SrFe}_{2} \mathrm{As}_{2}$ is $\boldsymbol{q}=\left(\begin{array}{lll}1 & 0 & 1\end{array}\right)$, thus the AFM coupling is realized in the longer $\mathrm{Fe}-\mathrm{Fe}$ direction within the Fe-As layer. The stacking along the $c$ direction is also AFM. The direction of the magnetic moment is parallel to the $a$ 
axis as well. A remarkable agreement was obtained in the temperature evolution of the magnetic moment and structural distortion obtained from independent measurements. These facts clearly demonstrate the close relationship between magnetism and lattice distortion in $\mathrm{SrFe}_{2} \mathrm{As}_{2}$.

The details on the sample preparation of $\mathrm{SrFe}_{2} \mathrm{As}_{2}$ have been described in Ref. 9. Neutron powder-diffraction experiments were carried out on the two-axis diffractometer E6, installed at Helmholtz Center Berlin, Germany. A double focusing pyrolytic graphite (PG) monochromator results in a high neutron flux at the sample position. Data were recorded at scattering angle up to $110^{\circ}$ using a two-dimensional position sensitive detector (2D PSD) with the size of 300 $\times 300 \mathrm{~mm}^{2}$. Simultaneous use of the double focusing monochromator and 2D PSD in combination with the radial oscillating collimator gives a high efficiency for taking diffraction patterns. The neutron wavelength was chosen to be $2.4 \AA$ in connection with a PG filter in order to avoid higher-order contaminations. As a compensation for the high efficiency, errors in the absolute values in the lattice constants become relatively large. Since the detailed absolute values were already obtained from the $\mathrm{x}$-ray diffraction, ${ }^{11}$ we relied on these data and focus here on the details of the magnetic structure. Fine powder of $\mathrm{SrFe}_{2} \mathrm{As}_{2}$ with a total mass of $\sim 2 \mathrm{~g}$ was sealed in a vanadium cylinder as sample container. The standard ${ }^{4} \mathrm{He}$ cryostat was used to cool the sample down to $1.5 \mathrm{~K}$ well below $T_{0}$. Neutron-diffraction patterns were taken at different temperatures between 1.5 and $220 \mathrm{~K}$ and the obtained diffraction patterns were analyzed by the Rietveld method using the software RIETAN-FP. ${ }^{20}$ The software VESTA (Ref. 21) was used to draw both crystal and magnetic structures.

Figure 1 shows neutron-diffraction patterns of $\mathrm{SrFe}_{2} \mathrm{As}_{2}$ taken at (a) $T=220 \mathrm{~K}\left(T>T_{0}\right)$ and (b) $T=1.5 \mathrm{~K}\left(T<T_{0}\right)$. Results of the Rietveld analysis, the residual intensity curve, and tick marks indicating the expected reflection angle are also plotted in the figure. For the high temperature $T>T_{0}$, the observed pattern is well reproduced by assuming the crystal structure with the space group $14 / \mathrm{mmm}$ as shown in Fig. 1(a). These results are consistent with that reported from $\mathrm{x}$-ray diffraction study, including the positional parameter of As, $z_{\mathrm{As}}=0.3602(2)$, where the number in the parenthesis indicates the uncertainty at the last decimal point. The conventional reliability factors $R_{\mathrm{wp}}=3.73 \%, R_{I}=3.18 \%$, and $R_{F}$ $=1.93 \%$ of the present analysis indicate the high quality of the present analysis. Small impurity peaks observed at around $63^{\circ}$ and $84^{\circ}$, which originate from $\mathrm{Cu}$ and $\mathrm{Al}$, were excluded from the analysis.

Below $T_{0}=205 \mathrm{~K}$, some nuclear Bragg reflections became broad. The inset of Fig. 1 shows the Bragg peak profile around $57.5^{\circ}$ where the 112 Bragg peak of the high temperature tetragonal phase was observed. The peak intensity drops and broadens on passing through $T_{0}$, as expected for the orthorhombic distortion where the lattice constant $a$ becomes longer than $b$. The reflection profile could be well reproduced by the lattice distortion from the tetragonal to orthorhombic lattice reported by the x-ray diffraction study. ${ }^{11}$ Within the errors, the positional parameter of As in the orthorhombic phase $z_{\mathrm{As}}=0.3604(2)$ is the same as that above $T_{0}$.

In addition to the lattice distortion, additional reflections

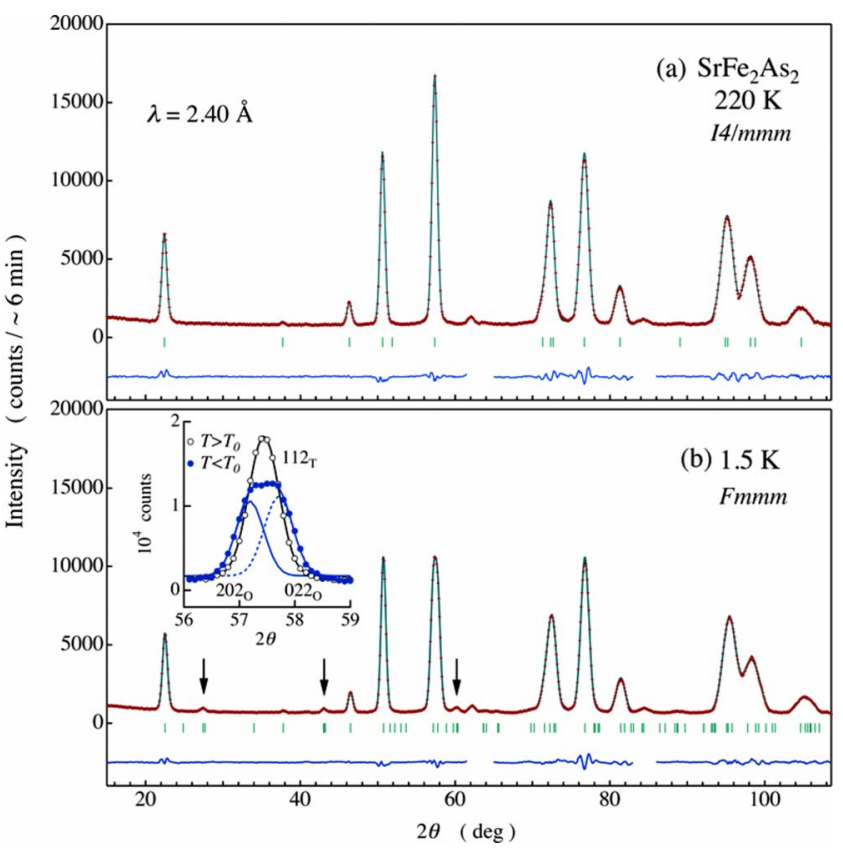

FIG. 1. (Color online) Neutron powder-diffraction patterns measured on E6 at (a) $220 \mathrm{~K}\left(T>T_{0}\right)$ and (b) $1.5 \mathrm{~K}\left(T<T_{0}\right)$. The result of the Rietveld analysis, residual curves, and the calculated peak positions indicated by tick marks are included in each panel. The inset shows the temperature variation in the peak profile around $2 \theta=57.5^{\circ}$.

were also observed at $T=1.5 \mathrm{~K}$ as indicated by arrows in Fig. 1(b). These superlattice peaks are most prominent at low scattering angles, and the corresponding peaks were not observed in the $\mathrm{x}$-ray diffraction. ${ }^{10,11}$ Therefore, the origin of these superlattice peaks should be magnetic. Figure 2 shows the temperature dependence of the integrated intensity of the superlattice reflection around $43^{\circ}$ which can be indexed as 103 as will be described later. The left axis is set to be proportional to the size of the magnetic moment, $\sqrt{I / I_{0}}$, where $I_{0}$ is the intensity at the lowest temperature. The 103 reflection appears below $T_{0}=205 \mathrm{~K}$ and shows a sharp increase in its intensity, which is clearly seen in the profile shown in the inset. The magnetic moment at $201 \mathrm{~K}$, just $4 \mathrm{~K}$ below $T_{0}$, already attains $70 \%$ of that at $1.5 \mathrm{~K}$. These findings strongly support the first-order transition at $T_{0}$ in $\mathrm{SrFe}_{2} \mathrm{As}_{2}$. In the same figure, the relative lattice distortion and the muon precession frequency of $\mathrm{SrFe}_{2} \mathrm{As}_{2}$ taken from Ref. 11 both normalized to the saturated values are plotted. A remarkable agreement of the temperature dependences is seen for these quantities obtained from independent measurements. This clearly demonstrates the close relationship between magnetism and lattice distortion in $\mathrm{SrFe}_{2} \mathrm{As}_{2}$.

Hereafter, we analyze the magnetic structure of $\mathrm{SrFe}_{2} \mathrm{As}_{2}$. For simplicity, in the magnetic structure analysis the structural parameters were fixed to the best value determined from the nuclear Bragg peaks. In the following, three representative models shown in the right panel of Fig. 3 are considered. At first, the direction of the AFM coupling with respect to the orthogonal axis is examined. This corresponds to the difference between model I with the propagation vector $\boldsymbol{q}=\left(\begin{array}{lll}1 & 0 & 1\end{array}\right)$ and model II with $\boldsymbol{q}=\left(\begin{array}{lll}0 & 1 & 1\end{array}\right)$. The difference 


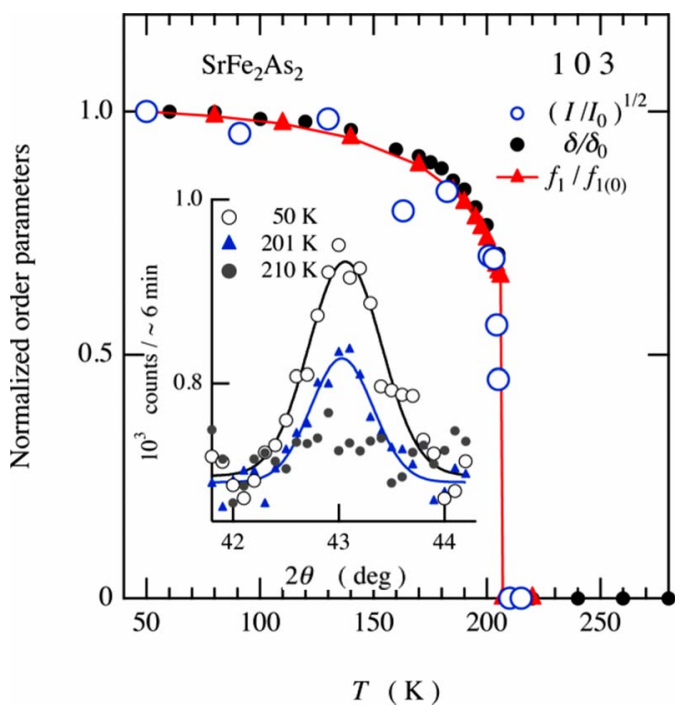

FIG. 2. (Color online) Temperature dependence of the 103 magnetic reflection intensity normalized to that at $1.5 \mathrm{~K}$, plotted as $\sqrt{I / I_{0}}$, which is proportional to the size of the ordered moment. Closed circles and triangles correspond to the temperature dependence of normalized lattice distortion and the muon precession frequency $f_{1}$, respectively, of $\mathrm{SrFe}_{2} \mathrm{As}_{2}$ taken from Ref. 11. The inset shows the 103 magnetic peak profile taken at 50, 201, and $210 \mathrm{~K}$.

between these models appears in the scattering angle arising from the subtle difference between $a$ and $b$. The difference can be clearly seen in the comparison for 101 (upper panel) and 121 (bottom) reflections. When model I is used as a

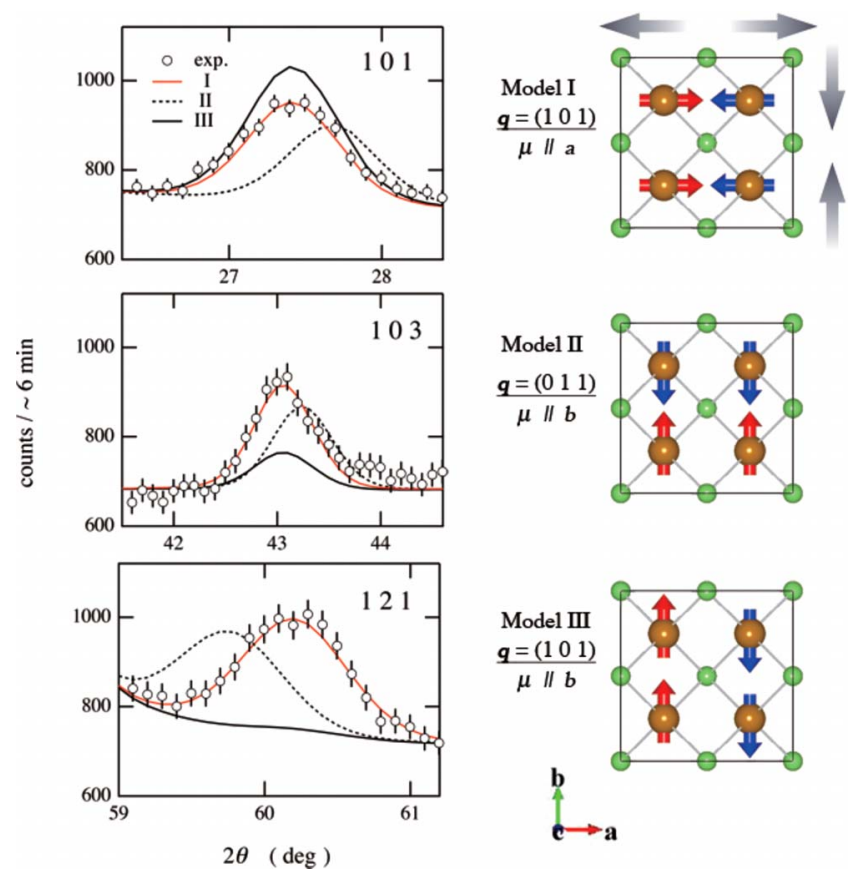

FIG. 3. (Color online) Magnetic Bragg peak profiles at (llll),

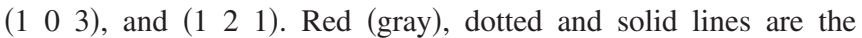
calculated intensity assuming the respective magnetic structure model: (i) $\boldsymbol{q}=\left(\begin{array}{lll}1 & 0 & 1\end{array}\right), \quad \mu \| a$, (ii) $\boldsymbol{q}=\left(\begin{array}{lll}0 & 1 & 1\end{array}\right), \mu \| b$, (iii) $\boldsymbol{q}$ $=\left(\begin{array}{lll}1 & 0 & 1\end{array}\right), \mu \| b$. Shaded arrows illustrate the orthorhombic distortion concomitantly occurs at $T_{0}$.

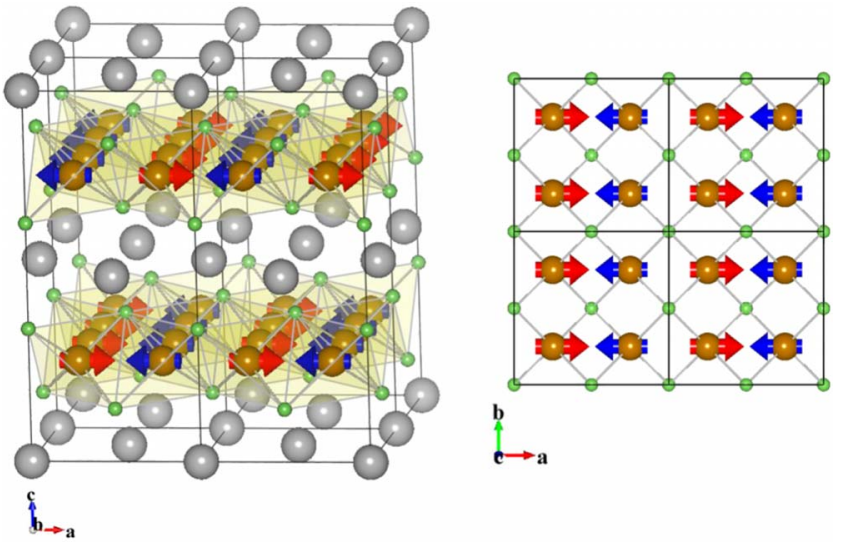

FIG. 4. (Color online) Magnetic and crystal structure of $\mathrm{SrFe}_{2} \mathrm{As}_{2}$ in the ground state. The AFM coupling is along the $a$ direction having the longer Fe-Fe distance, corresponding to $\boldsymbol{q}$ $=\left(\begin{array}{lll}1 & 0 & 1\end{array}\right)$. The Fe magnetic moment lies within the Fe plane and is aligned parallel to the $a$ axis.

reference, model II gives a higher scattering angle for 1001 which then corresponds to 011 , and a lower angle for 121 (2 11 1). The comparison in Fig. 3 clearly shows that only model I gives the correct positions of the 101 and 121 reflections. Therefore, the magnetic propagation vector of $\mathrm{SrFe}_{2} \mathrm{As}_{2}$ is determined to be $\boldsymbol{q}=\left(\begin{array}{lll}1 & 0 & 1\end{array}\right)$.

In a second step, we try to analyze the direction of AFM moment, which corresponds to model I and III. In model I, the magnetic moment is set to be parallel to the AFM coupling in the Fe-As plane, $\mu \| a$, whereas it is perpendicular in model III. The magnetic diffraction intensity depends on the angle between the magnetic moment and scattering vector. The powder average of this angle factor for the orthorhombic symmetry is given as follows:

$$
\left\langle q^{2}\right\rangle=1-\left(h^{2} a^{* 2} \cos ^{2} \psi_{a}+k^{2} b^{* 2} \cos ^{2} \psi_{b}+l^{2} c^{* 2} \cos ^{2} \psi_{c}\right) d^{2},
$$

where $h, k, l$ are the reflection indexes, $a^{*}, b^{*}, c^{*}$ are the primitive lattice vectors in reciprocal space, and $d$ is the spacing of the $(h k l)$ in real space. $\psi_{a}, \psi_{b}, \psi_{c}$ are the angles between the magnetic moment and crystallographic axes $a$, $b$, and $c$. This factor affects the relative magnetic intensities of 101,103 , and 121 diffraction peaks. Model I gives the comparable intensity for all three peaks, in good agreement with experimental results. In contrary, model III results in a strong 101 and a vanishing 121 peak, which does not at all fit with the measured intensities. As a result, the calculation based on model I gives an excellent agreement for the position and intensity of all magnetic reflections. Therefore, we can conclusively determine the direction of the magnetic moment to be $a$. By using the structure model I, the size of the Fe magnetic moment is deduced to be 1.01(3) $\mu_{B}$ with good reliability factors $R_{\mathrm{wp}}=3.54 \%, R_{I}=2.49 \%$, and $R_{F}=1.63 \%$. The determined magnetic structure of $\mathrm{SrFe}_{2} \mathrm{As}_{2}$ is shown in Fig. 4; the AFM coupling occurs along the longer $a$ direction and the moment orients in the same direction. The magnetic ordering in $\mathrm{SrFe}_{2} \mathrm{As}_{2}$ within the $\mathrm{FeAs}$ layer seems identical to that in $\mathrm{BaFe}_{2} \mathrm{As}_{2},{ }^{16}$ in $\operatorname{LaFeAs}(\mathrm{O}, \mathrm{F}),{ }^{14}$ and in 
$\mathrm{CeFeAs}(\mathrm{O}, \mathrm{F}),{ }^{15}$ although the stacking order is not common. This supports the importance of the FeAs intralayer coupling as well as the weak interlayer coupling.

We now turn to the discussion of the relation between the structural distortion and the magnetic structure. As mentioned before, the magnetic order in the FeAs system occurs either after the structural distortion or simultaneously. A simple approach would be to consider a superexchange path between the nearest Fe neighbors. Since the positional parameter of As and the lattice constant $c$ does not exhibit significant change, both the packing of the Fe-As layer and the Fe-As distance stay constant on passing through $T_{0}$. Thus, the distortion within the Fe-As layer at $T_{0}$ mainly results in a slight change in the Fe-Fe distance and the FeAs-Fe bond angle. The longer $a$ lattice constant leads to a slightly smaller bond angle along $a, \angle(\mathrm{Fe}-\mathrm{As}-\mathrm{Fe})_{a}=71.2^{\circ}$, as compared to that along $b, \angle(\mathrm{Fe}-\mathrm{As}-\mathrm{Fe})_{b}=72.1^{\circ}$; in other words, the difference is less than $1^{\circ}$. It is unlikely that such minor distortions lead to significant differences in exchange parameters. In contrast, it should be noted that the observed orthorhombic distortion and the columnar magnetic structure in $\mathrm{SrFe}_{2} \mathrm{As}_{2}$ are consistent with the results of a bandstructure calculation. ${ }^{11}$ The calculation gives both a comparable distortion and the correct magnetic structure in which the AFM arrangement is along the long $a$ axis. These calculations predict the distortion to be stable only for the columnar state, not for other magnetic structures or the nonmagnetic state, indicating the strong coupling of magnetic order and orthorhombic distortion. Similar results were also obtained for LaFeAsO. ${ }^{17-19}$ For this compound, Yildirim ${ }^{17}$ discusses the lattice distortion to be related to a lifting of the double degeneracy of the columnar state within a localized spin model for a frustrated square lattice. A detailed neutron scattering study on single crystals is expected to give further insights into this fascinating coupling.

The size of the magnetic moment of $1.01 \mu_{B}$ determined by the present neutron-diffraction study is the largest among the $R \mathrm{FeAsO}$ and the ternary arsenide $A \mathrm{Fe}_{2} \mathrm{As}_{2}$ reported so far: $0.36 \mu_{B}$ for $\operatorname{LaFeAs}(\mathrm{O}, \mathrm{F}),{ }^{14} 0.8 \mu_{B}$ for $\operatorname{CeFeAs}(\mathrm{O}, \mathrm{F}),{ }^{15}$ and $0.87 \mu_{B}$ for $\mathrm{BaFe}_{2} \mathrm{As}_{2}$. ${ }^{16}$ This corresponds to the stronger magnetism deduced for $\mathrm{SrFe}_{2} \mathrm{As}_{2}$ from bulk measurements, i.e., from the higher $T_{0}$ and the larger hyperfine field in Mössbauer experiments. On the other hand, it should be noted that the size of the ordered moment obtained in our neutron-diffraction study is almost twice as large as that deduced in other microscopic measurements, $\mu \mathrm{SR}$ and Mössbauer spectroscopy. Since the neutron-diffraction intensity is proportional to the square of the size of the moment, this large difference can hardly be attributed to an experimental error. Similar differences were obtained for $\mathrm{BaFe}_{2} \mathrm{As}_{2}$ and $R \mathrm{FeAsO}$ as well. ${ }^{13,22}$ This points to a general problem. While the neutrons directly probe the density of the magnetic moment, Mössbauer and $\mu$ SR rely on a scaling of the observed quantity, i.e., precession frequency and the hyperfine field. It might be that these scaling procedures, which are well established for stable magnetic Fe systems with large moment, are not appropriate for the unusual magnetism in these layered FeAs systems.

In conclusion, we have revealed the magnetic structure of $\mathrm{SrFe}_{2} \mathrm{As}_{2}$ by neutron powder diffraction. The magnetic propagation vector of $\mathrm{SrFe}_{2} \mathrm{As}_{2}$ is determined to be $\boldsymbol{q}$ $=\left(\begin{array}{lll}1 & 0 & 1\end{array}\right)$; the coupling of Fe moments is antiferromagnetic along both the longer $a$ direction within the Fe-As layer and the $c$ direction. The Fe magnetic moment with the size of 1.01(3) $\mu_{B}$ orients parallel to the $a$ axis. The temperature dependence of the magnetic moment shows excellent agreement with those of the muon precession frequency and the structural distortion, revealing the strong coupling of the spin-density-wave order and the structural distortion in $\mathrm{SrFe}_{2} \mathrm{As}_{2}$.

Note added. Single-crystal neutron study by Zhao et al. ${ }^{23}$ also concluded that the magnetic structure of $\mathrm{SrFe}_{2} \mathrm{As}_{2}$ has an antiferromagnetic coupling along the $a$ axis with an orientation parallel to the $a$ axis.

We thank H.-H. Klaus and H. Rosner for stimulating discussions.

\footnotetext{
*kaneko.koji@gmail.com

${ }^{1}$ Y. Kamihara, T. Watanabe, M. Hirano, and H. Hosono, J. Am. Chem. Soc. 130, 3296 (2008).

${ }^{2}$ Z.-A. Ren et al., Mater. Res. Innovations 12, 105 (2008).

${ }^{3}$ Z.-A. Ren et al., Europhys. Lett. 82, 57002 (2008).

${ }^{4}$ G. F. Chen, Z. Li, D. Wu, G. Li, W. Z. Hu, J. Dong, P. Zheng, J. L. Luo, and N. L. Wang, Phys. Rev. Lett. 100, 247002 (2008).

${ }^{5}$ X. H. Chen, T. Wu, G. Wu, R. H. Liu, H. Chen, and D. F. Fang, Nature (London) 453, 761 (2008).

${ }^{6}$ J. Yang et al., Supercond. Sci. Technol. 21, 082001 (2008).

${ }^{7}$ K. Sasmal, B. Lv, B. Lorenz, A. M. Guloy, F. Chen, Y. Y. Xue, and C. W. Chu, Phys. Rev. Lett. 101, 107007 (2008).

${ }^{8}$ G. F. Chen et al., Chin. Phys. Lett. 25, 3403 (2008).

${ }^{9}$ C. Krellner, N. Caroca-Canales, A. Jesche, H. Rosner, A. Ormeci, and C. Geibel, Phys. Rev. B 78, 100504(R) (2008).

${ }^{10}$ M. Tegel, M. Rotter, V. Weiss, F. M. Schappacher, R. Poettgen, and D. Johrendt, arXiv:0806.4782 (unpublished).
}

${ }^{11}$ A. Jesche et al., Phys. Rev. B 78, 180504(R) (2008).

${ }^{12}$ J.-Q. Yan et al., Phys. Rev. B 78, 024516 (2008).

${ }^{13}$ M. Rotter, M. Tegel, D. Johrendt, I. Schellenberg, W. Hermes, and R. Pottgen, Phys. Rev. B 78, 020503(R) (2008).

${ }^{14}$ C. de la Cruz et al., Nature (London) 453, 899 (2008).

${ }^{15}$ J. Zhao et al., Nature Mater. 7, 953 (2008).

${ }^{16}$ Q. Huang et al., arXiv:0806.2776 (unpublished).

${ }^{17}$ T. Yildirim, Phys. Rev. Lett. 101, 057010 (2008).

${ }^{18}$ P. V. Sushko, A. L. Shluger, M. Hirano, and H. Hosono, arXiv:0807.2213 (unpublished).

${ }^{19}$ I. I. Mazin, M. D. Johannes, L. Boeri, K. Koepernik, and D. J. Singh, arXiv:0806.1869 (unpublished).

${ }^{20}$ F. Izumi and K. Momma, Solid State Phenom. 130, 15 (2007).

${ }^{21}$ K. Momma and F. Izumi, J. Appl. Crystallogr. 41, 653 (2008).

${ }^{22}$ H.-H. Klauss et al., Phys. Rev. Lett. 101, 077005 (2008).

${ }^{23}$ J. Zhao, W. Ratcliff II, J. W. Lynn, G. F. Chen, J. L. Luo, N. L. Wang, J. Hu, and P. Dai, Phys. Rev. B 78, 140504(R) (2008). 